\title{
Does Tinnitus Depend on Time-of-Day? An Ecological Momentary Assessment Study with the "Track YourTinnitus" Application
}

\section{OPEN ACCESS}

Edited by:

Christopher R. Cederroth, Karolinska Institutet, Sweden

Reviewed by:

Karl Bechter,

University of Ulm, Germany

Robert D. Frisina

University of South Florida,

United States

*Correspondence:

Thomas Probst

thomas.probst@donau-uni.ac.at

${ }^{\dagger}$ These authors shared senior-authorship.

Received: 07 January 2017 Accepted: 17 July 2017 Published: 02 August 2017

Citation:

Probst $T$, Pryss RC, Langguth $B$

Rauschecker JP, Schobel J, Reichert M, Spiliopoulou M, Schlee W

and Zimmermann J (2017) Does

Tinnitus Depend on Time-of-Day? An Ecological Momentary Assessment

Study with the "TrackYourTinnitus" Application.

Front. Aging Neurosci. 9:253. doi: 10.3389/fnagi.2017.00253

\begin{abstract}
Thomas Probst ${ }^{1,2 *}$, Rüdiger C. Pryss' ${ }^{2}$, Berthold Langguth ${ }^{3}$, Josef P. Rauschecker ${ }^{4,5}$, Johannes Schobel ${ }^{2}$, Manfred Reichert ${ }^{2}$, Myra Spiliopoulou $^{6}$, Winfried Schlee $^{3 \dagger}$ and Johannes Zimmermann ${ }^{7 t}$

${ }^{1}$ Georg-Elias-Müller-Institute for Psychology, Georg-August-University Göttingen, Göttingen, Germany, ${ }^{2}$ Department for Psychotherapy and Biopsychosocial Health, Danube University Krems, Krems an der Donau, Austria, ${ }^{3}$ Department of Psychiatry and Psychotherapy of the University of Regensburg at Bezirksklinikum Regensburg, Regensburg, Germany, ${ }^{4}$ Program in Cognitive and Computational Systems, Georgetown University Washington, Washington, DC, United States, ${ }^{5}$ Institute for Advanced Study, Technical University Munich, Munich, Germany, ${ }^{6}$ Department of Technical and Business Information Systems, Otto-von-Guericke-University Magdeburg, Magdeburg, Germany, ${ }^{7}$ Psychologische Hochschule Berlin, Berlin, Germany
\end{abstract}

Only few previous studies used ecological momentary assessments to explore the time-of-day-dependence of tinnitus. The present study used data from the mobile application "TrackYourTinnitus" to explore whether tinnitus loudness and tinnitus distress fluctuate within a 24-h interval. Multilevel models were performed to account for the nested structure of assessments (level 1: 17,209 daily life assessments) nested within days (level 2: 3,570 days with at least three completed assessments), and days nested within participants (level 3: 350 participants). Results revealed a time-of-day-dependence of tinnitus. In particular, tinnitus was perceived as louder and more distressing during the night and early morning hours (from 12 a.m. to 8 a.m.) than during the upcoming day. Since previous studies suggested that stress (and stress-associated hormones) show a circadian rhythm and this might influence the time-of-day-dependence of tinnitus, we evaluated whether the described results change when statistically controlling for subjectively reported stress-levels. Correcting for subjective stress-levels, however, did not change the result that tinnitus (loudness and distress) was most severe at night and early morning. These results show that time-of-day contributes to the level of both tinnitus loudness and tinnitus distress. Possible implications of our results for the clinical management of tinnitus are that tailoring the timing of therapeutic interventions to the circadian rhythm of individual patients (chronotherapy) might be promising.

Keywords: tinnitus, stress, circadian fluctuation, time-of-day, ecological momentary assessment

\section{INTRODUCTION}

Tinnitus, the phantom perception of sound (Baguley et al., 2013; Langguth et al., 2013), is perceived by $5.1 \%$ up to $42.7 \%$ of the population according to a recent review including 39 studies from 16 countries (McCormack et al., 2016). These percentages depend on age (with older persons showing higher prevalence), gender (with male persons showing higher prevalence), and the definition of 
tinnitus used in the epidemiological study (McCormack et al., 2016). Prevalence rates of tinnitus also have increased over the years (Nondahl et al., 2012; Martinez et al., 2015), and one can speculate about the reasons for this apparent increase. On a neuronal basis, auditory-limbic interactions play a central role in the development of (chronic) tinnitus (Rauschecker et al., 2010; Leaver et al., 2011). In many cases, tinnitus is associated with psychological distress and incapacity for work (Bhatt et al., 2016) resulting in high socio-economic costs (Maes et al., 2013). Cognitive-behavioral therapy (CBT) has proven to have the potential to reduce the burden of tinnitus for the individual (Hesser et al., 2011) as well as the society/economy (Maes et al., 2014). But not all tinnitus patients reach clinically relevant improvements with CBT (e.g., Jasper et al., 2014). Other therapeutic approaches, including pharmacological therapy (Langguth and Elgoyhen, 2012), auditory stimulation (Hobson et al., 2012) or brain stimulation (Langguth and De Ridder, 2013), have only revealed small and inconsistent effects in subgroups of patients. It is assumed that an important reason for the poor treatment response in clinical trials is the heterogeneity of tinnitus (Landgrebe et al., 2012; Baguley et al., 2013), both across patients (inter-individual heterogeneity) as well as within patients over time (intra-individual heterogeneity, see Dauman et al., 2015).

Therefore, it is important to understand the factors that contribute to the heterogeneity of tinnitus. Psychological variables, such as fear-related cognition (e.g., Cima et al., 2011; Kleinstäuber et al., 2013), an accepting stance toward tinnitus (e.g., Weise et al., 2013; Riedl et al., 2015), emotions (e.g., Probst et al., 2016a,b), and avoidance/safety behaviors (e.g., Hesser and Andersson, 2009; Kleinstäuber et al., 2013), have been demonstrated to account for the heterogeneity of tinnitus in several studies (see also the "scientific cognitivebehavioral model of tinnitus"; McKenna et al., 2014). Moreover, recent neuroscience studies imply that the moment-to-moment variability of tinnitus is related to brain oscillatory patterns like the alpha power in temporal regions (Schlee et al., 2014) and time-of-day (Basinou et al., 2017); circadian fluctuations have been shown, for example, in auditory pathway structures related to tinnitus like the cochlea (Meltser et al., 2014) and the inferior colliculus (Park et al., 2016). Another hint for a link between tinnitus and circadian rhythms is provided by findings of reduced tinnitus severity after intake of melatonin (e.g., Pirodda et al., 2010; Ajayi et al., 2014; Miroddi et al., 2015). Furthermore, pain, which shares many similarities with tinnitus (De Ridder et al., 2011; Rauschecker et al., 2015), has been found to underlie circadian variations (e.g., Strian et al., 1989; Gilron and Ghasemlou, 2014; Buttgereit et al., 2015). Furthermore, depression, which overlaps in its pathophysiology with tinnitus (Langguth et al., 2011), is characterized by changes in circadian rhythm (e.g., Germain and Kupfer, 2008; Wirz-Justice, 2008).

The question of whether tinnitus varies systematically over the course of the day, however, has not yet been studied systematically. The limited possibilities of traditional assessment methods to routinely track symptoms in the daily routine have made it rather difficult to validly study whether tinnitus shows time-of-day-dependence. To overcome these limitations of traditional assessment methods, newer technological developments can be used to electronically gather valid daily life data (ecological momentary assessments, EMA; Trull and Ebner-Priemer, 2013, 2014; Adams et al., 2017). Henry et al. (2012), for example, used personal digital assistants (PDA) in a 2-week pilot study with 24 participants to obtain EMA during a 12-h interval (8 a.m.-8 p.m.) and showed that the scores of the 10-item screening version of the Tinnitus Handicap Inventory (THI-S; Newman et al., 2008) were not significantly different between 3-h time blocks ( 8 a.m.-11 a.m., 11 a.m.-2 p.m., 2 p.m.5 p.m., and 5 p.m. -8 p.m.). Although Henry et al. (2012) failed to demonstrate time-of-day-dependence of tinnitus, a 2-week pilot smartphone-based study with 20 participants on fluctuations of tinnitus within an 11-h interval (9 a.m.-8 p.m.) suggested that tinnitus does vary within a single day (Wilson et al., 2015). But Wilson et al. (2015) did not report at which time-of-day the participants rated their tinnitus as more or less severe. In another diary study, Flor et al. (2004) reported tinnitus being worst at the beginning of a day, thus supporting the time-of-daydependence of tinnitus. To our knowledge there are only these three studies with ambivalent results that addressed the time-ofday-dependence of tinnitus in daily life with EMA (Flor et al., 2004; Henry et al., 2012; Wilson et al., 2015).

The current study used EMA from the "TrackYourTinnitus" (TYT) mobile application (Pryss et al., 2015a,b; Schlee et al., 2016) to explore whether tinnitus fluctuates within a 24-h interval (night and upcoming day). Tinnitus is operationalized by two questions in TYT, one question is on tinnitus loudness and the other question on tinnitus distress. Prior research suggested that an assessment of both tinnitus loudness and tinnitus distress is necessary for a comprehensive assessment, since tinnitus loudness and tinnitus distress are only moderately correlated (e.g., Hiller and Goebel, 2007; Wallhäusser-Franke et al., 2012) and processed in different but interconnected brain areas (e.g., Leaver et al., 2012; Ueyama et al., 2013; De Ridder et al., 2014; Vanneste et al., 2014). Therefore, it appears possible that tinnitus loudness and tinnitus distress show either similar or different ups and downs within a 24-h interval. Accordingly, the present study investigated the timeof-day-dependence of tinnitus loudness as well as of tinnitus distress. Moreover, stress is known to be associated with tinnitus (e.g., Hébert et al., 2004; Hébert and Lupien, 2007, 2009; Alsalman et al., 2016) and stress-related hormones like cortisol and adrenocorticotropic hormone (ACTH) underlie circadian rhythms (e.g., Dickmeis, 2009; Lightman and Conway-Campbell, 2010; Conway-Campbell et al., 2012), which could influence the potential time-of-day-dependence of tinnitus (loudness and distress). Thus, we also explored whether the stress-level as assessed with TYT depends on time-of-day and whether the 24$\mathrm{h}$ fluctuations of tinnitus loudness and tinnitus distress change when taking the stress-level into account.

\section{MATERIALS AND METHODS}

The material and the methods were approved by the Ethics Committee of the University Clinic of Regensburg and were 
carried out in accordance with the approved guidelines. Information that the TYT data will be used for scientific analyses is included in the mobile applications of "TrackYourTinnitus" as well as on the "TrackYourTinnitus" website and, therefore, the TYT users were informed that the data will be used for scientific purposes. Written consent, however, was not possible to obtain given the nature of the study. The study participants were anonymized.

\section{"TrackYourTinnitus" Platform}

The TYT platform (www.trackyourtinnitus.org, Pryss et al., 2015a,b) consists of a website for registration, two mobile applications (for iOS and Android), and a MySQL database as a central repository for the data collected. Users can either use TYT whenever they want or they can set a user-defined schedule to receive random notifications. For the study at hand, only these notification-triggered assessments were investigated. At each of these notifications, the users are asked to rate their tinnitus and other tinnitus-related variables (e.g., subjective stress-level). Although the attention might be directed toward the tinnitus by such notifications, Henry et al. (2012) and Schlee et al. (2016) found that repeatedly rating tinnitus and associated variables in daily life does not have detrimental effects. The present study investigated the following variables the users were asked to rate at each notification: "Current tinnitus loudness" (subjective rating of current tinnitus loudness on a visual analog scale [VAS], including a zero value for moments without loudness: min: 0; max: 1), "current tinnitus distress" (subjective rating of current tinnitus distress on a VAS including a zero value for moments without distress: min: 0; $\max : 1$ ), and "current stresslevel" (subjective rating of current stress-level on a VAS: min: 0; max: 1). Moreover, the timestamps of the assessments were used to explore the time-of-day-dependence of tinnitus. In TYT, the timestamps represent the local time of the time zone a given user is in when providing the assessments.

The data set used for the current study was exported in June 2016. After excluding the self-initiated assessments and the assessments given within the $15 \mathrm{~min}$ after the last assessment (for the inter-assessment interval of $15 \mathrm{~min}$ see also Pryss et al., 2015b), we had access to 25,863 notification-triggered assessments. For the present study, we only included the 25,092 assessments without missing values in any of the three target variables. Furthermore, as we were interested in within-day variations, we only considered data from days with at least three completed assessments, resulting in a total number of 17,209 assessments.

\section{Sample}

The final sample consisted of 350 participants. Two-hundred and fifty three participants (72.2\%) were male, 94 (26.9\%) were female, and 3 did not indicate their gender. On average, participants were $45.4(S D=12.1)$ years old (17 participants did not report their age). The median number of years since onset of tinnitus was 5.4, ranging from 0 to 61.8 years. According to participants, onset of tinnitus was related to loud blast of sound $(n=48)$, whiplash $(n=9)$, change in hearing $(n=38)$, stress $(n=99)$, head trauma $(n=12)$, and other causes $(n=$
141) (3 participants did not report events related to onset of tinnitus). The median number of days per participant (with at least three assessments) was 11, ranging from 1 to 415 days. This corresponds to a total number of 3,570 days. The median number of assessments per day was 4, ranging from 3 (the minimum requirement to be included in this study) to 18 assessments.

\section{Statistical Analyses}

To test our hypotheses, we used multilevel modeling (MLM; Raudenbush and Bryk, 2002; Singer and Willett, 2003). MLM is ideally suited to address the nested structure of our data, with assessments (Level 1) nested in days (Level 2), and days nested in participants (Level 3). First, we estimated two MLMs predicting tinnitus loudness and tinnitus distress from timeof-day, respectively. Time-of-day was dummy-coded using five binary variables indicating whether the assessment was in the early morning (T1, from 4 a.m. to 8 a.m.), in the late morning (T2, from 8 a.m. to 12 p.m.), in the afternoon (T3, from 12 p.m. to 4 p.m.), in the early evening (T4, from 4 p.m. to 8 p.m.), or in the late evening (T5, from 8 p.m. to 12 a.m.). Assessments during the night (from 12 a.m. to 4 a.m.) were defined as the reference group. The full three-level MLM with random intercepts at Level 2 and 3 and random slopes at Level 3 (Model I) is summarized below:

Level 1: $\quad y_{i j k}=\pi_{0 j k}+\pi_{1 j k}(T 1)+\pi_{2 j k}(T 2)+\pi_{3 j k}(T 3)$

Level 2: $\quad \pi_{0 j k}=\beta_{00 k}+r_{0 j k}$ $+\pi_{4 j k}(T 4)+\pi_{5 j k}(T 5)+e_{i j k}$

$\pi_{1 j k}=\beta_{10 k}$

$\pi_{2 j k}=\beta_{20 k}$

$\pi_{3 j k}=\beta_{30 k}$

$\pi_{4 j k}=\beta_{40 k}$

$\pi_{5 j k}=\beta_{50 k}$

Level 3: $\quad \beta_{00 k}=\gamma_{000}+u_{00 k}$

$\beta_{10 k}=\gamma_{100}+u_{10 k}$

$\beta_{20 k}=\gamma_{200}+u_{20 k}$

$\beta_{30 k}=\gamma_{300}+u_{30 k}$

$\beta_{40 k}=\gamma_{400}+u_{40 k}$

$\beta_{50 k}=\gamma_{500}+u_{50 k}$

The model decomposes the amount of tinnitus loudness/distress (y) of participant $k$ on day $j$ at assessment $i$ into a series of fixed and random effects. The fixed effect $\gamma_{000}$ represents the expected (population) tinnitus loudness/distress during the night before an average day of an average participant. The fixed effects $\gamma_{100}, \gamma_{200}, \gamma_{300}, \gamma_{400}$, and $\gamma_{500}$ represent the expected change in tinnitus loudness/distress from night to early and late morning, afternoon and early and late evening, respectively. The random effects at Level $3, u_{00 k}, u_{10 k}, u_{20 k}, u_{30 k}, u_{40 k}$, and $u_{50 k}$, indicate that the level of tinnitus loudness/distress at night as well as its later change during the day may differ between participants. The random intercept at Level 2, $r_{0 j k}$, indicates that the baseline level of tinnitus loudness/distress may differ between days within participants. We assumed random effects to be multivariate normally distributed within levels, and residuals to be independent and identically distributed across levels. 
Second, we estimated the same MLM for stress-level as the dependent variable (y). Moreover, we estimated two further MLMs predicting tinnitus loudness and tinnitus distress from time-of-day, this time including stress-level as an additional predictor. As stress-level varied across all three levels, we decomposed its variance into three separate mean-centered variables capturing variation of stress within days (S1), variation of stress within participants across days (S2), and variation of stress across participants (S3) prior to estimating the MLMs. The full three-level MLM with random intercepts at Level 2 and 3 and random slopes at Level 3 (Model II) is summarized below:

$$
\begin{aligned}
& \text { Level 1: } \quad y_{i j k}=\pi_{0 j k}+\pi_{1 j k}(T 1)+\pi_{2 j k}(T 2)+\pi_{3 j k}(T 3) \\
& +\pi_{4 j k}(T 4)+\pi_{5 j k}(T 5)+\pi_{6 j k}(S 1)+e_{i j k} \\
& \text { Level 2: } \quad \pi_{0 j k}=\beta_{00 k}+\beta_{01 k}(S 2)+r_{0 j k} \\
& \pi_{1 j k}=\beta_{10 k} \\
& \pi_{2 j k}=\beta_{20 k} \\
& \pi_{3 j k}=\beta_{30 k} \\
& \pi_{4 j k}=\beta_{40 k} \\
& \pi_{5 j k}=\beta_{50 k} \\
& \pi_{6 j k}=\beta_{60 k}
\end{aligned}
$$

The newly defined fixed effects, $\gamma_{001}, \gamma_{010}$, and $\gamma_{600}$, represent the expected between-participant, between-day, and within-day effect of stress on tinnitus loudness/distress after controlling for time-of-day. The remaining fixed effects, $\gamma_{000}, \gamma_{100}, \gamma_{200}$, $\gamma_{300}, \gamma_{400}$, and $\gamma_{500}$, represent the expected level of tinnitus loudness/distress at night as well as its later change during the day after controlling for the influence of stress. The newly defined random effects, $u_{01 k}$ and $u_{60 k}$, indicate that the betweenand within-day effects of stress may differ between participants. Due to model identification issues, we restricted the covariances between random effects of stress and the remaining random effects in the model to be zero.

All models were estimated using full maximum likelihood estimation. Analyses were conducted with the package "lme4" (Bates et al., 2015) of the statistical platform R (R Core Team, 2015). We used Satterthwaite's approximations to derive p-values for fixed effects. Pairwise comparisons between the six distinct timeframes were explored using the Tukey Honest Significant Difference method as implemented in the package "multcomp" (Hothorn et al., 2008). Finally, we quantified the effect size of time-of-day on tinnitus loudness/distress by means of a pseudo $R^{2}$ statistic. This statistic can be computed by subtracting the residual variance $\operatorname{Var}\left(e_{i j k}\right)$ of Model I from the residual variance of an intercept-only model without any predictors, divided by this latter residual variance. It represents the relative amount of variance in tinnitus loudness/distress within days that is explained by time-of-day (i.e., by the five variables T1-T5).

\section{RESULTS}

In total, 186 assessments (1.1\%) were completed at night, $460(2.7 \%)$ were completed in the early morning, 4,200 (24.4\%) were completed in the late morning, 4,941 (28.7\%) in the afternoon, $4,724(27.5 \%)$ in the early evening, and $2,698(15.7 \%)$ were completed in the late evening (see Figure 1). Table 1 summarizes the estimated fixed effects of all MLMs (standard deviations and correlations of random effects can be found in the Supplementary Material). Results suggest that tinnitus was louder and more distressing during the night and early morning hours than during all other timeframes of the day (see Models I in the first and third columns of Table 1). Tukey's post-hoc tests revealed that differences between late morning, afternoon, and early evening were non-significant (see Table 2). However, tinnitus was significantly louder in the late evening compared to the afternoon and early evening. This pattern of results is visualized in Figures 2A,B. The pseudo $\mathrm{R}^{2}$ statistics revealed that time-ofday explained $20.6 \%$ of the within-day variance of tinnitus loudness, and $13.0 \%$ of the within-day variance of tinnitus distress.

Next, we tested whether time-of-day influences the subjective stress-level (see Model I in the right hand column of Table 1 and Figure 2C). Tukey's post-hoc tests suggested that stress-level increased from morning to afternoon, decreased from afternoon to evening, and did not differ compared to the night (see Table 2). Time-of-day explained $7.8 \%$ of the within-day variance of stress.

Finally, the models predicting tinnitus loudness and tinnitus distress from time-of-day and stress-level revealed that the stress-level had incremental effects across all three levels (see Models II in Table 1): Tinnitus was louder and more distressing when the level of stress was higher at a specific time-ofday compared to other times-of-day, when it was higher during a whole day compared to other days, and when it was higher during the whole assessment period for a given participant (compared to other participants). Nevertheless, the effects of time-of-day on tinnitus loudness and tinnitus distress

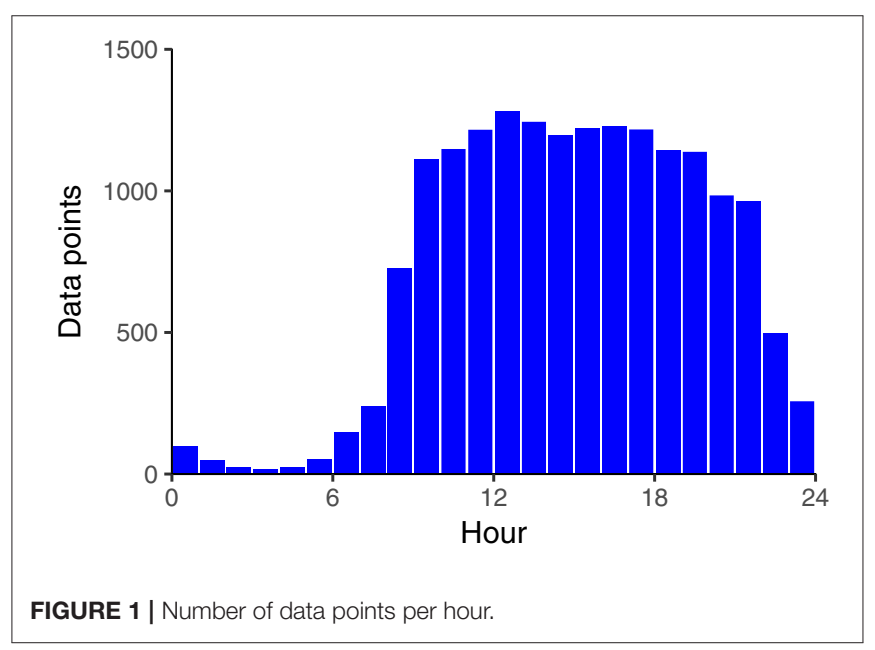


TABLE 1 | Estimated fixed effects (and their standard errors) for five MLMs.

\begin{tabular}{|c|c|c|c|c|c|c|}
\hline & & \multicolumn{2}{|c|}{ Tinnitus loudness } & \multicolumn{2}{|c|}{ Tinnitus distress } & \multirow{2}{*}{$\frac{\text { Stress-level }}{\text { Model I }}$} \\
\hline & & Model I & Model II & Model I & Model II & \\
\hline Intercept (night) & $\gamma 000$ & $0.529^{\star \star \star}(0.021)$ & $0.521^{\star \star \star}(0.018)$ & $0.435^{\star \star \star}(0.021)$ & $0.423^{\star \star \star}(0.016)$ & $0.303^{\star \star \star}(0.020)$ \\
\hline Early morning (vs. night) effect & $\gamma_{100}$ & $-0.017(0.021)$ & $-0.015(0.019)$ & $-0.015(0.022)$ & $-0.014(0.018)$ & $-0.015(0.020)$ \\
\hline Late morning (vs. night) effect & $\gamma_{200}$ & $-0.089^{\star \star \star}(0.019)$ & $-0.086^{\star \star \star}(0.017)$ & $-0.083^{\star \star \star}(0.020)$ & $-0.077^{\star \star \star}(0.016)$ & $0.009(0.019)$ \\
\hline Afternoon (vs. night) effect & $\gamma_{300}$ & $-0.098^{\star \star \star}(0.019)$ & $-0.099^{\star \star \star}(0.017)$ & $-0.083^{\star \star \star}(0.019)$ & $-0.083^{\star \star \star}(0.016)$ & $0.023(0.018)$ \\
\hline Early evening (vs. night) effect & $\gamma_{400}$ & $-0.093^{\star \star \star}(0.019)$ & $-0.092^{\star \star \star}(0.017)$ & $-0.080^{\star \star \star}(0.020)$ & $-0.074^{\star \star \star}(0.015)$ & $0.006(0.018)$ \\
\hline Late evening (vs. night) effect & $\gamma_{500}$ & $-0.071^{\star \star \star}(0.019)$ & $-0.062^{\star \star \star}(0.016)$ & $-0.066^{\star \star \star}(0.019)$ & $-0.052^{\star \star \star}(0.014)$ & $-0.023(0.018)$ \\
\hline Within-day effect of stress & $\gamma_{600}$ & & $0.287^{\star \star \star}(0.020)$ & & $0.370^{\star \star \star}(0.022)$ & \\
\hline Between-day effect of stress & $\gamma_{010}$ & & $0.470^{\star \star \star}(0.037)$ & & $0.585^{\star \star \star}(0.035)$ & \\
\hline Between-person effect of stress & $\gamma_{001}$ & & $0.651^{\star \star \star}(0.051)$ & & $0.810^{\star \star \star}(0.041)$ & \\
\hline
\end{tabular}

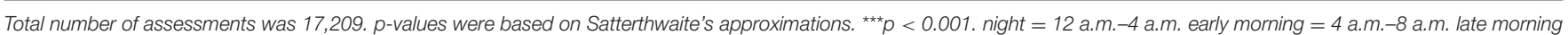
$=8$ a.m.-12 p.m. afternoon $=12$ p.m.-4 p.m. early evening $=4$ p.m. -8 p.m. late evening $=8$ p.m. -12 a.m.

TABLE 2 | Tukey's post-hoc tests for five MLMs.

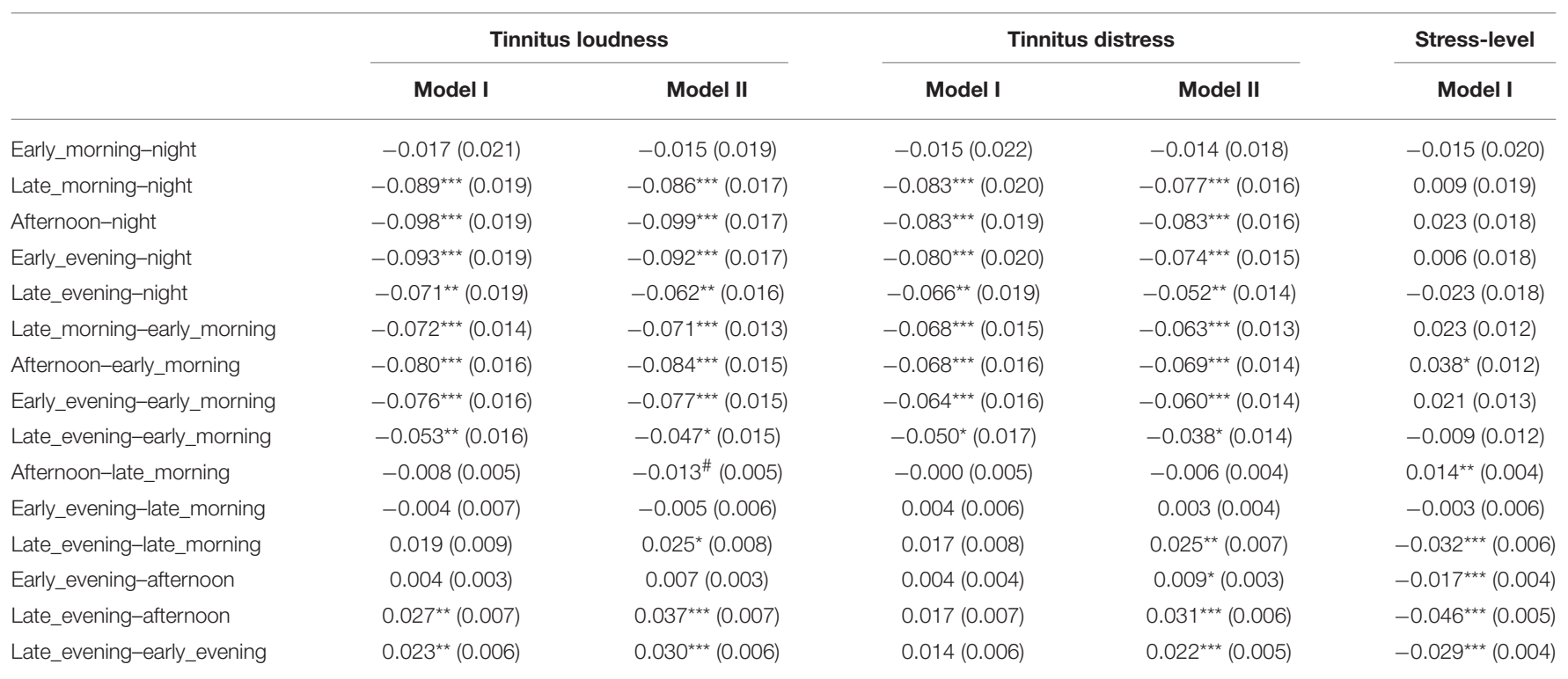

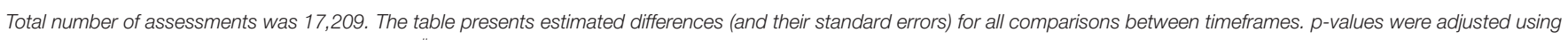


afternoon $=12$ p.m. -4 p.m. early evening $=4$ p.m. -8 p.m. late evening $=8$ p.m. -12 a.m.

were still significant (i.e., after controlling for the effects of stress).

\section{DISCUSSION}

This study evaluated whether subjective tinnitus loudness and subjective tinnitus distress depend on time-of-day with EMA from the "TrackYourTinnitus" (TYT) mobile application (Pryss et al., 2015a,b; Schlee et al., 2016). Strengths of the present study are that a mobile application was used to obtain EMA of high ecological validity (Trull and Ebner-Priemer, 2013, 2014; Adams et al., 2017), a much higher sampling frequency was applied than in typical clinical studies, and the sample size was much larger than in previous EMA studies on the time-of-day-dependence of tinnitus (Flor et al., 2004; Henry et al., 2012; Wilson et al., 2015).
The main result was that tinnitus (loudness and distress) was rated as more severe during the night and the early morning (from 12 a.m. to 8 a.m.) than during the upcoming day. Interestingly, tinnitus loudness and tinnitus distress showed a very similar time pattern although the neurobiological correlates of loudness and distress differ to a certain degree (e.g., De Ridder et al., 2011; Leaver et al., 2012; Ueyama et al., 2013; Vanneste et al., 2014).

Contrary to the EMA study by Henry et al. (2012), the present investigation found a time-of-day-dependence of tinnitus. The most obvious reason for this discrepancy is that Henry et al. (2012) assessed tinnitus only from 8 a.m. to 8 p.m. and could, therefore, not evaluate tinnitus during the time interval that was related to most severe tinnitus in our study (12 a.m.-8 a.m.). During a time interval ( 8 a.m.-12 a.m.) that included the $12-\mathrm{h}$ 

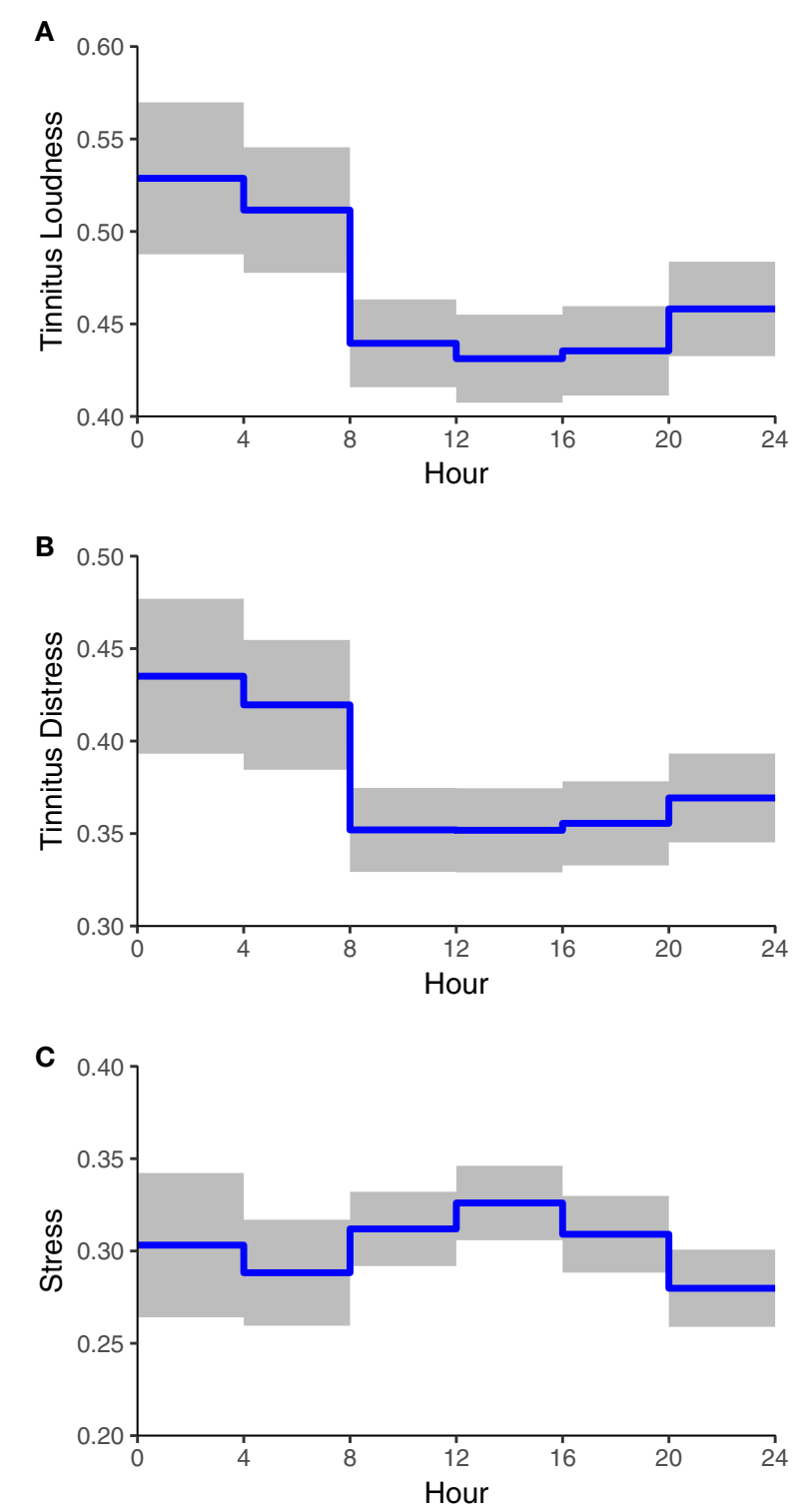

FIGURE 2 | Expected tinnitus loudness (A), tinnitus distress (B), and level of stress (C) as a function of time-of-day. The blue lines represent expected values for the average participant on an average day, the gray bands represent 95\% confidence intervals.

interval analyzed by Henry et al. (2012) tinnitus did not vary much in our study either (see Figures 2A,B). The result that tinnitus was more severe in the early morning hours is in line with a previous diary study (Flor et al., 2004) and fits to the clinical impression of a "morning roar" as it is often anecdotally reported by tinnitus patients. Yet, our result that tinnitus was more severe during the night was not found by Flor et al. (2004). More severe tinnitus (loudness and distress) during the night could at least partially result from the possibility that several assessments at night were given by participants while having sleep disturbances. Sleep disturbances are common among tinnitus patients and their severity correlates positively with measures of tinnitus severity (e.g., Crönlein et al., 2007; Schecklmann et al.,
2015; Crönlein et al., 2016). Although we aimed to control for this potential confounder by excluding data from spontaneous ratings and restricting the data analysis to the ratings provided at notifications, the fact that sleep disturbances were not assessed on a daily basis in the present study and, hence, were not available for analysis is a limitation of our study. Future research could explore whether the effect of time-of-day on tinnitus is different between days with and without sleep disturbances. Besides sleep, several other variables are time-of-day dependent and should be considered as potential confounding variables: For example, the environmental sound level depends on time-of-day and tinnitus might be more severe during the night and the early morning, because the environmental sound level in these time intervals cannot mask the tinnitus. Furthermore, a trigger of tinnitus might be (even slight) tension of specific muscles of the craniocervical connection (Bechter et al., 2016), whereby the tension depends mainly from posture, which is influenced by different aspects during the night (e.g., sleeping posture, bed pillow) and during the day. Moreover, the release of stress-related hormones is controlled by a circadian clock (Dickmeis, 2009; Lightman and Conway-Campbell, 2010; Conway-Campbell et al., 2012) and future studies could explore the relationship between circadian tinnitus variability and such stress-related hormones. In the present study, we could only analyze subjective stresslevels with the result that the 24-h course of the subjective stresslevel was not parallel to the course of tinnitus (loudness and distress): While tinnitus (loudness and distress) showed their maximum between 12 a.m. and 8 a.m., the maximum of the subjective stress-level was found between 12 p.m. and 4 p.m. The discrepant time curves of tinnitus and subjective stresslevel raise further questions, which need to be addressed in future longitudinal studies regarding the tinnitus-stress link. For example, it appears possible that tinnitus and subjective stress interact more in a time-lagged than in an instant manner. Thus, it should be clarified whether increases of subjective stress lead to more severe tinnitus and whether increases of tinnitus severity also lead to more subsequent subjective stress (which again might affect tinnitus severity resulting in a vicious circle). Feedback loops have been described already in research on the pituitaryadrenal system: "positive, delayed, feedforward connection between the pituitary and the adrenals" and "negative feedback of glucocorticoids on ACTH release" (Lightman and ConwayCampbell, 2010, p. 712). It should also be noted in this context that the peaks of our self-reported stress-levels (12 p.m. -4 p.m.) did not correspond to the peaks of cortisol and ACTH releases (4 a.m.-8 a.m.) as illustrated by Lightman and Conway-Campbell (2010). Therefore, the longitudinal associations between tinnitus and self-reported stress might be different from the ones between tinnitus and stress-hormones.

Regarding the observed circadian variability of tinnitus loudness, it is tempting to speculate relations to circadian variations in neuronal sensitivity of peripheral and central auditory structures, which have been identified in recent studies (Park et al., 2016; Basinou et al., 2017). However, tinnitus loudness does not directly reflect neuronal activity in auditory pathways, but also depends on other factors such as attention and emotions. For example, a previous study found that tinnitus becomes louder over time when participants experience 
more qualitatively different feelings (Probst et al., 2016b). Therefore, more research is needed to replicate the observed circadian rhythm of tinnitus in large samples, to identify the specific contribution of different factors (e.g., neuronal activity in auditory pathways, attention, and emotion), and to explore whether different persons show a different time-of-day dependence of tinnitus. Moderating variables might, for example, be gender, age (e.g., the time-of-day dependence of tinnitus might be different between men and women or within women before/after the menopause), and the etiology of tinnitus (e.g., the time of time-of-day dependence might be different between noise trauma as etiology and stress as etiology). Future translational and clinical studies with samples large enough to analyze such subgroups are required to explore potential moderators of the time-of-day dependence of tinnitus. These further studies could also measure tinnitus by different assessment methods, because the study at hand only analyzed self-reported tinnitus. For example, by the "gap-in-noise" paradigm (Lowe and Walton, 2015) or psychophysiological measurements such as tinnitus matching. Yet, the reliability and validity of tinnitus matching is an ongoing matter of debate (Hoare et al., 2014; De Ridder et al., 2015). For mobile applications such as TYT, portable methods to assess tinnitus more objectively (see for example, Hébert and Fournier, 2017) that can be integrated in the mobile application are needed. It is necessary to evaluate whether a similar timeof-day dependence of tinnitus can be shown for self-reported tinnitus as well as for more objectively measured tinnitus. Future studies could also compare different statistical approaches and identify the approach most suited to investigate time-of-day dependencies. For example, harmonic regression was performed in an animal study on circadian rhythm (Atger et al., 2015), and an $\mathrm{R}$ package for nonparametric circular methods (NPCirc) is available to analyze circular data (Oliveira et al., 2014). These approaches were not performed in the present study due to the nested structure of the data (assessments within days and days within participants).

In summary, the results of our study have implications for both tinnitus research and tinnitus management. Taking timeof-day into account in the study design might be necessary in clinical studies. Yet, the results of our study rely solely on TYT users (who are not representative for clinical tinnitus patients, see Probst et al., 2017) and on one-item questions on tinnitus distress and tinnitus loudness making it difficult to conclude whether the observed differences between night/early morning and the upcoming day are clinically relevant. Future studies using assessment instruments appropriate to distinguish between clinically relevant and clinically irrelevant change (for example the Tinnitus Handicap Inventory (THI), see Zeman et al., 2011) with clinical tinnitus samples are necessary to address this

\section{REFERENCES}

Adams, W. Z., McClure, E. A., Gray, K. M., Danielson, C. K., Treiber, F. A., and Ruggiero, K. J. (2017). Mobile devices for the remote acquisition of physiological and behavioral biomarkers in psychiatric point. Chronobiological aspects should not only be considered in tinnitus research but also in tinnitus treatment. Tailoring the timing of therapeutic interventions to the circadian rhythm of individual tinnitus patients (chronotherapy) might be promising. Further translational and clinical studies are necessary to evaluate the potential of chronotherapy for tinnitus. However, this is a challenging task since "research in the topic is underfunded when compared with other diseases for which the prevalence and cost to society is relatively similar" (Cederroth et al., 2013, p. 972). Nevertheless, the current study might motivate individuals with tinnitus to observe their tinnitus fluctuations and to identify time-intervals during which the access to and the use of tinnitus coping strategies are most crucial.

\section{AUTHOR CONTRIBUTIONS}

TP substantially contributed to the design of the study and data preparation, drafted and revised the manuscript. RP substantially contributed to the design of the study, data preparation, conception, implementation and maintenance of the "TrackYourTinnitus" application, and revised the manuscript. BL substantially contributed to the design of the study and revised the manuscript. JR substantially contributed to the design of the study and revised the manuscript. IS substantially contributed to the conception, implementation and maintenance of the "TrackYourTinnitus" application, and revised the manuscript. MR substantially contributed to the conception, implementation and maintenance of the "TrackYourTinnitus" application, and revised the manuscript. MS substantially contributed to the design of the study and revised the manuscript. WS substantially contributed to the design of the study, data preparation, conception and implementation of the "TrackYourTinnitus" application, drafted and revised the manuscript. JZ substantially contributed to the design of the study and performed the statistical analysis, drafted and revised the manuscript.

\section{ACKNOWLEDGMENTS}

This work was supported by the German Research Foundation (DFG) and the Georg-August-University Göttingen (Germany) within the funding programme Open Access Publishing.

\section{SUPPLEMENTARY MATERIAL}

The Supplementary Material for this article can be found online at: http://journal.frontiersin.org/article/10.3389/fnagi. 2017.00253/full\#supplementary-material 
Alsalman, O. A., Tucker, D., and Vanneste, S. (2016). Salivary stress-related responses in tinnitus: a preliminary study in young male subjects with tinnitus. Front. Neurosci. 10:338. doi: 10.3389/fnins.2016.00338

Atger, F., Gobet, C., Marquis, J., Martin, E., Wang, J., Weger, B., et al. (2015). Circadian and feeding rhythms differentially affect rhythmic mRNA transcription and translation in mouse liver. Proc. Natl. Acad. Sci. U.S.A. 112, E6579-E6588. doi: 10.1073/pnas.1515308112

Baguley, D., McFerran, D., and Hall, D. (2013). Tinnitus. Lancet 382, 1600-1607. doi: 10.1016/S0140-6736(13)60142-7

Basinou, V., Park, J.- S., Cederroth, C. R., and Canlon, B: (2017). Circadian regulation of auditory function. Hear. Res. 347, 47-55. doi: 10.1016/j.heares.2016.08.018

Bates, D., Maechler, M., Bolker, B, and Walker, S. (2015). Fitting linear mixedeffects models using lme4. J. Stat. Softw. 67, 1-48. doi: 10.18637/jss.v067.i01

Bechter, K., Wieland, M., and Hamann, G. F. (2016). Chronic cervicogenic tinnitus rapidly resolved by intermittent use of cervical collar. Front. Psychiatry 7:43. doi: 10.3389/fpsyt.2016.00043

Bhatt, J. M., Bhattacharyya, N., and Lin, H. W. (2016). Relationships between tinnitus and the prevalence of anxiety and depression. Laryngoscope 127, 466-469. doi: 10.1002/lary.26107

Buttgereit, F., Smolen, J. S., Coogan, A. N., and Cajochen, C. (2015). Clocking in: chronobiology in rheumatoid arthritis. Nat. Rev. Rheumatol. 11, 349-356. doi: 10.1038/nrrheum.2015.31

Cederroth, C. R., Canlon, B., and Langguth, B. (2013). Hearing loss and tinnitus-are funders and industry listening? Nat. Biotechnol. 31, 972-974. doi: $10.1038 /$ nbt. 2736

Cima, R. F., Crombez, G., and Vlaeyen, J. W. (2011). Catastrophizing and fear of tinnitus predict quality of life in patients with chronic tinnitus. Ear. Hear. 32, 634-641. doi: 10.1097/AUD.0b013e31821106dd

Conway-Campbell, B. L., Pooley, J. R., Hager, G. L., and Lightman, S. L. (2012). Molecular dynamics of ultradian glucocorticoid receptor action. Mol. Cell. Endocrinol. 348, 383-393. doi: 10.1016/j.mce.2011.08.014

Crönlein, T., Langguth, B., Geisler, P., and Hajak, G. (2007). Tinnitus and insomnia. Prog. Brain. Res. 166, 227-233. doi: 10.1016/S0079-6123(07)66021-X

Crönlein, T., Langguth, B., Pregler, M., Kreuzer, P. M., Wetter, T. C., and Schecklmann, M. (2016). Insomnia in patients with chronic tinnitus: cognitive and emotional distress as moderator variables. J. Psychosom. Res. 83, 65-68. doi: 10.1016/j.jpsychores.2016.03.001

Dauman, N., Erlandsson, S., Lundlin, L., and Dauman, R. (2015). Intra-individual variability in tinnitus patients: current thoughts and perspectives. HNO. 63, 302-306. doi: 10.1007/s00106-014-2978-2

De Ridder, D., Congedo, M., and Vanneste, S. (2015). The neural correlates of subjectively perceived and passively matched loudness perception in auditory phantom perception. Brain Behav. 5: e00331. doi: 10.1002/ brb3.331

De Ridder, D., Elgoyhen, A. B., Romo, R., and Langguth, B. (2011). Phantom percepts: tinnitus and pain as persisting aversive memory networks. Proc. Natl. Acad. Sci. U.S.A. 108, 8075-8080. doi: 10.1073/pnas.1018466108

De Ridder, D., Vanneste, S., Weisz, N., Londero, A., Schlee, W., Elgoyhen, A. B., et al. (2014). An integrative model of auditory phantom perception: tinnitus as a unified percept of interacting separable subnetworks. Neurosci. Biobehav. Rev. 44, 16-32. doi: 10.1016/j.neubiorev.2013.03.021

Dickmeis, T. (2009). Glucocorticoids and the circadian clock. J. Endocrinol. 200, 3-22. doi: 10.1677/JOE-08-0415

Flor, H., Hoffmann, D., Struve, M., and Diesch, E. (2004). Auditory discrimination training for the treatment of tinnitus. Appl. Psychophysiol. Biofeedback 29, 113-120. doi: 10.1023/B:APBI.0000026637.77671.f4

Germain, A., and Kupfer, D. J. (2008). Circadian rhythm disturbances in depression. Hum. Psychopharmacol. 23, 571-585. doi: 10.1002/hup.964

Gilron, I., and Ghasemlou, N. (2014). Chronobiology of chronic pain: focus on diurnal rhythmicity of neuropathic pain. Curr. Opin. Support. Palliat. Care 8, 429-436. doi: 10.1097/SPC.0000000000000085

Hébert, S., and Fournier, P. (2017). Clinical validation of a new tinnitus assessment technology. Front. Neurol. 8:38. doi: 10.3389/fneur.2017.00038

Hébert, S., and Lupien, S. J. (2007). The sound of stress: blunted cortisol reactivity to psychosocial stress in tinnitus sufferers. Neurosci. Lett. 411, 138-142. doi: 10.1016/j.neulet.2006.10.028

Hébert, S., and Lupien, S. J. (2009). Salivary cortisol levels, subjective stress, and tinnitus intensity in tinnitus sufferers during noise exposure in the laboratory. Int. J. Hyg. Environ. Health 212, 37-44. doi: 10.1016/j.ijheh.2007. 11.005

Hébert, S., Paiement, P., and Lupien, S. J. (2004). A physiological correlate for the intolerance to both internal and external sounds. Hear. Res. 190, 1-9. doi: 10.1016/S0378-5955(04)00021-8

Henry, J. A., Galvez, G., Turbin, M. B., Thielman, E. J., McMillan, G. P., and Istvan, J. A. (2012). Pilot study to evaluate ecological momentary assessment of tinnitus. Ear. Hear. 33, 179-290. doi: 10.1097/AUD.0b013e31822f6740

Hesser, H., and Andersson, G. (2009). The role of anxiety sensitivity and behavioral avoidance in tinnitus disability. Int. J. Audiol. 48, 295-299. doi: 10.1080/14992020802635325

Hesser, H., Weise, C., Westin, V. Z., and Andersson, G. (2011). A systematic review and meta-analysis of randomized controlled trials of cognitivebehavioral therapy for tinnitus distress. Clin. Psychol. Rev. 31, 545-553. doi: 10.1016/j.cpr.2010.12.006

Hiller, W., and Goebel, G. (2007). When tinnitus loudness and annoyance are discrepant: audiological characteristics and psychological profile. Audiol. Neurootol. 12, 391-400. doi: 10.1159/000106482

Hoare, D. J., Edmondson-Jones, M., Gander, P. E., and Hall, D. A. (2014). Agreement and reliability of tinnitus loudness matching and pitch likeness rating. PLoS ONE 9:e114553. doi: 10.1371/journal.pone.0114553

Hobson, J., Chisholm, E., and El Refaie, A. (2012). Sound therapy (masking) in the management of tinnitus in adults. Cochrane Database Syst. Rev. 11:CD006371. doi: 10.1002/14651858.cd006371.pub3

Hothorn, T., Bretz, F., and Westfall, P. (2008). Simultaneous inference in general parametric models. Biom. J. 50, 346-363. doi: 10.1002/bimj.200810425

Jasper, K., Weise, C., Conrad, I., Andersson, G., Hiller, W., and Kleinstäuber, M. (2014). Internet-based guided self-help versus group cognitive behavioral therapy for chronic tinnitus: a randomized controlled trial. Psychother. Psychosom. 83, 234-246. doi: 10.1159/000360705

Kleinstäuber, M., Jasper, K., Schweda, I., Hiller, W., Andersson, G., and Weise, C. (2013). The role of fear-avoidance cognitions and behaviors in patients with chronic tinnitus. Cogn. Beh. Ther. 42, 84-99. doi: 10.1080/16506073.2012.717301

Landgrebe, M., Azevedo, A., Baguley, D., Bauer, C., Cacace, A., Coelho, C., et al. (2012). Methodological aspects of clinical trials in tinnitus: a proposal for an international standard. J. Psychosom. Res. 73, 112-121. doi: 10.1016/j.jpsychores.2012.05.002

Langguth, B., and De Ridder, D. (2013). Tinnitus: therapeutic use of superficial brain stimulation. Handb. Clin. Neurol. 116, 441-467. doi: 10.1016/B978-0-444-53497-2.00036-X

Langguth, B., and Elgoyhen, A. B. (2012). Current pharmacological treatments for tinnitus. Expert Opin. Pharmacother. 13, 2495-2509. doi: $10.1517 / 14656566.2012 .739608$

Langguth, B., Kreuzer, P. M., Kleinjung, T., and De Ridder, D. (2013). Tinnitus: causes and clinical management. Lancet Neurol. 12, 920-930. doi: 10.1016/S1474-4422(13)70160-1

Langguth, B., Landgrebe, M., Kleinjung, T., Sand, G. P., and Hajak, G. (2011). Tinnitus and depression. World J. Biol. Psychiatry 12, 489-500. doi: 10.3109/15622975.2011.575178

Leaver, A. M., Renier, L., Chevillet, M. A., Morgan, S., Kim, H. J., and Rauschecker, J. P. (2011). Dysregulation of limbic and auditory networks in tinnitus. Neuron 69, 33-43. doi: 10.1016/j.neuron.2010.12.002

Leaver, A. M., Seydell-Greenwald, A., Turesky, T. K., Morgan, S., Kim, H. J., and Rauschecker, J. P. (2012). Cortico-limbic morphology separates tinnitus from tinnitus distress. Front. Syst. Neurosci. 6:21. doi: 10.3389/fnsys.2012.00021

Lightman, S. L., and Conway-Campbell, B. L. (2010). The crucial role of pulsatile activity of the HPA axis for continuous dynamic equilibration. Nat. Rev. Neurosci. 11, 710-718. doi: 10.1038/nrn2914

Lowe, A. S., and Walton, J. P. (2015). Alterations in peripheral and central components of the auditory brainstem response: a neural assay of tinnitus. PLoS ONE 10:e0117228. doi: 10.1371/journal.pone.0117228

Maes, I. H., Cima, R. F., Anteunis, L. J., Scheijen, D. J., Baguley, D. M., El Refaie, A., et al. (2014). Cost-effectiveness of specialized treatment based on cognitive behavioral therapy versus usual care for tinnitus. Otol. Neurotol. 35, 787-795. doi: 10.1097/MAO.0000000000000331

Maes, I. H., Cima, R. F., Vlaeyen, J. W., Anteunis, L. J., and Joore, M. A. (2013). Tinnitus: a cost study. Ear. Hear. 34, 508-514. doi: 10.1097/AUD.0b013e31827d113a 
Martinez, C., Wallenhorst, C., McFerran, D., and Hall, D. A. (2015). Incidence rates of clinically significant tinnitus: 10-year trend from a cohort study in England. Ear. Hear. 36, e69-75. doi: 10.1097/aud.0000000000000121

McCormack, A., Edmondson-Jones, M., Somerset, S, and Hall, D. (2016). A systematic review of the reporting of tinnitus prevalence and severity. Hear. Res. 337, 70-79. doi: 10.1016/j.heares.2016.05.009

McKenna, L., Handscomb, L., Hoare, D. J., and Hall, D. A. (2014). A scientific cognitive-behavioral model of tinnitus: novel conceptualizations of tinnitus distress. Front. Neurol. 5:196. doi: 10.3389/fneur.2014.00196

Meltser, I., Cederroth, C. R., Basinou, V., Savelyev, S., Lundkvist, G. S., and Canlon, B. (2014). TrkB-mediated protection against circadian sensitivity to noise trauma in the murine cochlea. Curr. Biol. 24, 658-663. doi: 10.1016/j.cub.2014.01.047

Miroddi, M., Bruno, R., Galletti, F., Calapai, F., Navarra, M., Gangemi, S., et al. (2015). Clinical pharmacology of melatonin in the treatment of tinnitus: a review. Eur. J. Clin. Pharmacol. 71, 263-270. doi: 10.1007/s00228-0151805-3

Newman, C. W., Sandridge, S. A., and Bolek, L. (2008). Development and psychometric adequacy of the screening version of the tinnitus handicap inventory. Otol. Neurotol. 29, 276-281. doi: 10.1097/MAO.0b013e31816569c4

Nondahl, D. M., Cruickshanks, K. J., Huang, G. H., Klein, B. E., Klein, R., Tweed, T. S., et al. (2012). Generational differences in the reporting of tinnitus. Ear. Hear. 33, 640-644. doi: 10.1097/AUD.0b013e31825069e8

Oliveira, M., Crujeiras, R. M., and Rodríguez-Casal, A. (2014). NPCirc: an R package for nonpara-metric circular methods. J. Stat. Soft. 61, 1-26. doi: $10.18637 /$ jss.v061.i09

Park, J., S., Cederroth, C. R., Basinou, V., Meltser, I., Lundkvist, G., and Canlon, B. (2016). Identification of a circadian clock in the inferior colliculus and its dysregulation by noise exposure. J. Neurosci. 36, 5509-5519. doi: 10.1523/JNEUROSCI.3616-15.2016

Pirodda, A., Raimondi, M. C., and Ferri, G. G. (2010). Exploring the reasons why melatonin can improve tinnitus. Med. Hypotheses 75, 190-191. doi: 10.1016/j.mehy.2010.02.018

Probst, T., Pryss, R., Langguth, B., and Schlee, W. (2016a). Emotional states as mediators between tinnitus loudness and tinnitus distress in daily life: results from the "TrackYourTinnitus" application. Sci. Rep. 6:20382. doi: $10.1038 /$ srep20382

Probst, T., Pryss, R., Langguth, B., and Schlee, W. (2016b). Emotion dynamics and tinnitus: daily life data from the "TrackYourTinnitus" application. Sci. Rep. 6:31166. doi: 10.1038/srep31166

Probst, T., Pryss, R. C., Langguth, B., Spiliopoulou, M., Landgrebe, M., Vesala, M., et al. (2017). Outpatient tinnitus clinic, self-help web platform, or mobile application to recruit tinnitus study samples? Front. Aging Neurosci. 9:113. doi: 10.3389/fnagi.2017.00113

Pryss, R., Reichert, M., Herrmann, J., Langguth, B., Schlee, W. (2015a). "Mobile crowd sensing in clinical and psychological trials - a case study," in 28th IEEE Int'l Symposium on Computer-Based Medical Systems, 22-25 June 2015 (Sao Carlos: IEEE Computer Society Press), 23-24. Available online at: http://dbis. eprints.uni-ulm.de/1144/1/PCBMS_2015.pdf (Accessed July 15, 2017)

Pryss, R., Reichert, M., Langguth, B., and Schlee, W. (2015b). "Mobile crowd sensing services for tinnitus assessment, therapy and research," in IEEE 4th International Conference on Mobile Services (MS 2015), June 27-July 2, 2015 (New York, NY: IEEE Computer Society Press), 352-359. Available online at: http://dbis.eprints.uni-ulm.de/1152/1/ms2015rpmrblws.pdf (Accessed: July 15, 2017).

Raudenbush, S. W., and Bryk, A. S. (2002). Hierarchical Linear Models: Applications and Data Analysis Methods. Newbury Park, CA: Sage Publications.

Rauschecker, J. P., Leaver, A. M., and Mühlau, M. (2010). Tuning out the noise: limbic-auditory interactions in tinnitus. Neuron 66, 819-826. doi: 10.1016/j.neuron.2010.04.032

Rauschecker, J. P., May, E. S., Maudoux, A., and Ploner, M. (2015). Frontostriatal gating of tinnitus and chronic pain. Trends Cogn. Sci. 19, 567-578. doi: 10.1016/j.tics.2015.08.002

R Core Team, A. (2015). R: A Language and Environment for Statistical Computing. Vienna: $\mathrm{R}$ foundation for statistical computing. Available online at: https:// www.R-project.org/ (Accessed: November 25, 2016).
Riedl, D., Rumpold, G., Schmidt, A., Zorowka, P. G., Bliem, H. R., and Moschen, R. (2015). The influence of tinnitus acceptance on the quality of life and psychological distress in patients with chronic tinnitus. Noise Health 17, 374-381. doi: 10.4103/1463-1741.165068

Schecklmann, M., Pregler, M., Kreuzer, P. M., Poeppl, T. B., Lehner, A., Crönlein, T., et al. (2015). Psychophysiological associations between chronic tinnitus and sleep: a cross validation of tinnitus and insomnia questionnaires. BioMed. Res. Int. 2015:461090. doi: 10.1155/2015/461090

Schlee, W., Pryss, R. C., Probst, T., Schobel, J., Bachmeier, A., Reichert, M., et al. (2016). Measuring the moment-to-moment variability of tinnitus: the TrackYourTinnitus smart phone app. Front. Aging Neurosci. 8:294. doi: 10.3389/fnagi.2016.00294

Schlee, W., Schecklmann, M., Lehner, A., Kreuzer, P. M., Vielsmeier, V., Poeppl, T. B., et al. (2014). Reduced variability of auditory alpha activity in chronic tinnitus. Neural. Plast. 2014:436146. doi: 10.1155/2014/436146

Singer, J. D., and Willett, J. B. (2003). Applied Longitudinal Data Analysis: Modeling Change and Event Occurence. New York, NY: Oxford University Press. doi: 10.1093/acprof:oso/9780195152968.001.0001

Strian, F., Lautenbacher, S., Galfe, G., and Hölzl, R. (1989). Diurnal variations in pain perception and thermal sensitivity. Pain 36, 125-131. doi: 10.1016/0304-3959(89)90120-6

Trull, T. J., and Ebner-Priemer, U. (2013). Ambulatory assessment. Annu. Rev. Clin. Psychol. 9, 151-176. doi: 10.1146/annurev-clinpsy-050212-185510

Trull, T. J., and Ebner-Priemer, U. (2014). The role of ambulatory assessment in psychological science. Curr. Dir. Psychol. Sci. 23, 466-470. doi: $10.1177 / 0963721414550706$

Ueyama, T., Donishi, T., Ukai, S., Ikeda, Y., Hotomi, M., Yamanaka, N., et al. (2013). Brain regions responsible for tinnitus distress and loudness: a resting-state FMRI study. PLoS ONE 8:e67778. doi: 10.1371/journal.pone.00 67778

Vanneste, S., Congedo, M., and De Ridder, D. (2014). Pinpointing a highly specific pathological functional connection that turns phantom sound into distress. Cereb. Cortex 24, 2268-2282. doi: 10.1093/cercor/bht068

Wallhäusser-Franke, E., Brade, J., Balkenhol, T., D'Amelio, R., Seegmüller, A., and Delb, W. (2012). Tinnitus: distinguishing between subjectively perceived loudness and tinnitus-related distress. PLOS ONE 7:e34583. doi: 10.1371/journal.pone.0034583

Weise, C., Kleinstäuber, M., Hesser, H., Westin, V. Z., and Andersson, G. (2013). Acceptance of tinnitus: validation of the tinnitus acceptance questionnaire. Cogn. Behav. Ther. 42, 100-115. doi: 10.1080/16506073.2013.781670

Wilson, M. B., Kallogjeri, D., Joplin, C. N., Gorman, M. D., Krings, J. G., Lenze, E. J., et al. (2015). Ecological momentary assessment of tinnitus using smartphone technology: a pilot study. Otolaryngol. Head. Neck. Surg. 152, 897-903. doi: $10.1177 / 0194599815569692$

Wirz-Justice, A. (2008). Diurnal variation of depressive symptoms. Dialogues Clin. Neurosci. 10, 337-343.

Zeman, F., Koller, M., Figueiredo, R., Aazevedo, A., Rates, M., Coelho, C., et al. (2011). Tinnitus handicap inventory for evaluating treatment effects: which changes are clinically relevant? Otolaryngol. Head Neck Surg. 145, 282-287. doi: $10.1177 / 0194599811403882$

Conflict of Interest Statement: The other authors declare that the research was conducted in the absence of any commercial or financial relationships that could be construed as a potential conflict of interest.

The handling Editor declared a co-authorship with the authors TP, RP, BL, MR, MS and WS, and the handling Editor states that the process met the standards of a fair and objective review.

Copyright (c) 2017 Probst, Pryss, Langguth, Rauschecker, Schobel, Reichert, Spiliopoulou, Schlee and Zimmermann. This is an open-access article distributed under the terms of the Creative Commons Attribution License (CC BY). The use, distribution or reproduction in other forums is permitted, provided the original author(s) or licensor are credited and that the original publication in this journal is cited, in accordance with accepted academic practice. No use, distribution or reproduction is permitted which does not comply with these terms. 